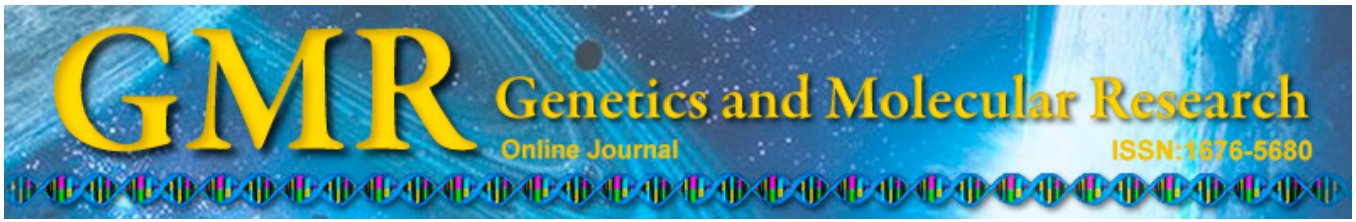

\title{
Analysis of signaling pathways in recurrent breast cancer
}

\author{
J.Z. Wu ${ }^{1}$, T.J. Yang ${ }^{2}$, P. Lu' ${ }^{2}$ and W. $\mathrm{Ma}^{3}$ \\ ${ }^{1}$ Breast Cancer Diagnosis and Treatment Center Henan, \\ The Affiliated Cancer Hospital of Zhengzhou University, Zhengzhou, China \\ ${ }^{2}$ Gastrointestinal Surgery Department, People's Hospital of Zhengzhou, \\ Zhengzhou, China \\ ${ }^{3}$ Department of Oncology, First Affiliated Hospital of Zhengzhou University, \\ Zhengzhou, China
}

Corresponding author: W. Ma

E-mail: linmeiwangzz@hotmail.com

Genet. Mol. Res. 13 (4): 10097-10104 (2014)

Received August 5, 2013

Accepted September 22, 2014

Published December 4, 2014

DOI http://dx.doi.org/10.4238/2014.December.4.4

\begin{abstract}
Breast cancer remains the second largest cause of death in women from cancer. By analyzing gene expression profiles in samples from breast cancer patients, 844 differentially expressed genes (DEGs) were identified in breast cancer metastasis. The 10 most significant signaling pathways identified through enrichment analysis contained DEGs were involved in oxidative phosphorylation, DNA replication, extracellular matrix-receptor interactions and others. Furthermore, survival analysis demonstrated that 5 of these signaling pathways were closely related to the survival time of breast cancer patients including basal transcription factors, cell cycle, ECM-receptor interaction, spliceosome, and DNA replication. Our findings increase the understanding of the network of signaling pathways involved in breast cancer metastasis and may provide theoretical support for further therapeutic study.
\end{abstract}

Key words: Breast cancer; Pathway; Survival analysis 


\section{INTRODUCTION}

Breast cancer is considered the leading cause of cancer-related deaths in women worldwide (Kamangar et al., 2006). Treatment options for metastatic breast cancer have improved with the increase in knowledge regarding connections between signaling pathways and biological behaviors. However, several major clinical and scientific issues remain unresolved, including the prevention, diagnosis, tumor progression and recurrence, treatment, and therapeutic resistance of breast cancer. There are 5 major molecular subtypes of breast cancer: basal-like, luminal A, luminal B, HER2+/ER-, and normal breast-like (Perou et al., 2000; Hu et al., 2006; Sørlie et al., 2006), which are conserved in different ethnic populations (Yu et al., 2004). Breast cancer is highly heterogeneous at both the molecular and clinical levels (Perou et al., 2000; Sørlie et al., 2001), which makes it difficult to cure and assess risk factors of metastasis.

Over the last decade, there has been an increasing in our understanding of the molecular mechanisms involved in breast cancer progression, as well as the crucial role of identifying genetic alterations for the early diagnosis and treatment of breast cancer. It is well known that among all populations, an estimated $5-10 \%$ of breast cancer cases arise in individuals with mutations in genes such as BRCA1 and BRCA2 (Claus et al., 1991; Easton et al., 1995; Schubert et al., 1997). Furthermore, recent advances in DNA microarray technology and other methods for large-scale gene expression analysis have been adopted for both biological characterization and therapeutic planning in breast cancer (Olopade et al., 2008). Understanding the molecular biology and gene expression signatures of breast cancer are critical for developing novel approaches toward prevention and therapy.

We conducted a bioinformatic analysis to investigate the pathological mechanism in primary breast cancer and to identify corresponding signaling pathways. Furthermore, through survival analysis, we identified biological pathways that were correlated to the survival time of breast cancer patients.

\section{MATERIAL AND METHODS}

\section{Gene expression profiles of breast cancer}

The transcription profile of GSE2034 (Wang et al., 2005) was obtained from the Gene Expression Omnibus (GEO, http://www.ncbi.nlm.nih.gov/geo/) database. This data set contains 180 samples from lymph node-negative primary breast cancer patients without recurrence and 106 samples from lymph node-negative primary breast cancer patients with metastasis. In addition, among the clinical data for these samples, survival time of each patient, defined as the time to relapse or to last follow-up, were collected. Based on the GPL96 [(HG-U133A) Affymetrix Human Genome U133A Array] dataset, RNA expression of primary breast cancer cells was analyzed. The clinical protocol was approved by the National Cancer Institute of China Institutional Review Board committee, and informed consent was obtained from all patients.

\section{Analysis of differentially expressed genes (DEGs)}

We compared the expression of genes in the lymph node-negative primary breast cancer patients without recurrence and with metastasis samples. Using the spatial analysis method (SAM), only DEGs with q-values less than 0.1 were selected. 


\section{Pathway analysis}

The Kyoto Encyclopedia of Genes and Genomes (KEGG) pathway database (Kanehisa and Goto, 2000) is a collection of manually drawn pathway maps of molecular interactions and reaction networks. Using SubpathwayMiner (Li et al., 2009), DEGs between the 2 sample types were enriched through the KEGG database in order to identify critical pathways involved in tumor metastasis. Pathways with false discovery rate (FDR) $<0.05$ were considered to be statistically significant.

\section{Survival analysis}

Pathways that we obtained were used to as biomarkers for survival analysis. The 286 breast cancers were clustered into 2 categories: class 1 and class 2 . Next, based on the survival time of these samples, survival curves for class 1 and class 2 were drawn. The log-rank test was used to compare the 2 survival curves and corresponding $\mathrm{P}$ values were determined.

\section{RESULTS}

\section{DEGs identification}

Based on GSE2034 data and the SAM approach, a total of 844 DEGs were identified in the lymph node-negative samples with metastasis comparing to without recurrence samples.

\section{Signaling pathways correlated with breast cancer metastasis}

Identified DEGs were subjected to KEGG pathway enrichment analysis, and were found to be enriched in various pathways. According to the P values and FDR values, the 10 most significant pathways (i.e., FDR $<0.05$ ) were collected (Table 1$)$. Among the pathways, the extracellular matrix $(\mathrm{ECM})$-receptor interaction $(\mathrm{P}=3.16 \mathrm{E}-05, \mathrm{FDR}=0.004953)$ and cell cycle $(\mathrm{P}=5.59 \mathrm{E}-05, \mathrm{FDR}=0.004953)$ pathways were the most significant. Moreover, DEGs were involved in oxidative phosphorylation, DNA replication, and proteasome pathways. In addition, we found that DEGs were also enriched in several signaling pathways related to Alzheimer's disease, Parkinson's disease, and Huntington's disease.

Table 1. Results of KEGG pathway enrichment analysis of 844 differentially expressed genes.

\begin{tabular}{llll}
\hline Pathway ID & Pathway name & P value & FDR \\
\hline path: 00190 & Oxidative phosphorylation & 0.000301 & 0.028406 \\
path: 03022 & Basal transcription factors & 0.000522 & 0.048059 \\
path: 03030 & DNA replication & $7.74 \mathrm{E}-05$ & 0.028406 \\
path: 03040 & Spliceosome & 0.002096 & 0.089964 \\
path: 03050 & Proteasome & 0.0004 & 0.028406 \\
path: 04110 & Cell cycle & $5.59 \mathrm{E}-05$ & 0.004953 \\
path: 04512 & ECM-receptor interaction & $3.16 \mathrm{E}-05$ & 0.004953 \\
path: 05010 & Alzheimer's disease & 0.001273 & 0.089964 \\
path: 05012 & Parkinson's disease & 0.000812 & 0.048059 \\
path: 05016 & Huntington's disease & 0.001207 & 0.048059 \\
\hline
\end{tabular}




\section{Signaling pathways correlated with the survival time of breast cancer patients}

After identifying the biological pathways related to breast cancer recurrence, we further analyzed the pathways correlated with survival time. Based on the expression levels of genes involved in these pathways, we distinguished samples with long survival time from those with short survival time. Therefore, all genes in the 10 obtained significant pathways were considered as biomarkers and were adopted for survival analysis (Figures 1-5). P values of 5 signaling pathways were less than 0.1 , including basal transcription factors (survival $\mathrm{P}$ $=0.00209$ ), cell cycle, ECM-receptor interaction, spliceosome, and DNA replication (Table 2) pathways. Pathways with lower P values indicate that those are more closely related to the survival time of breast cancer patients.

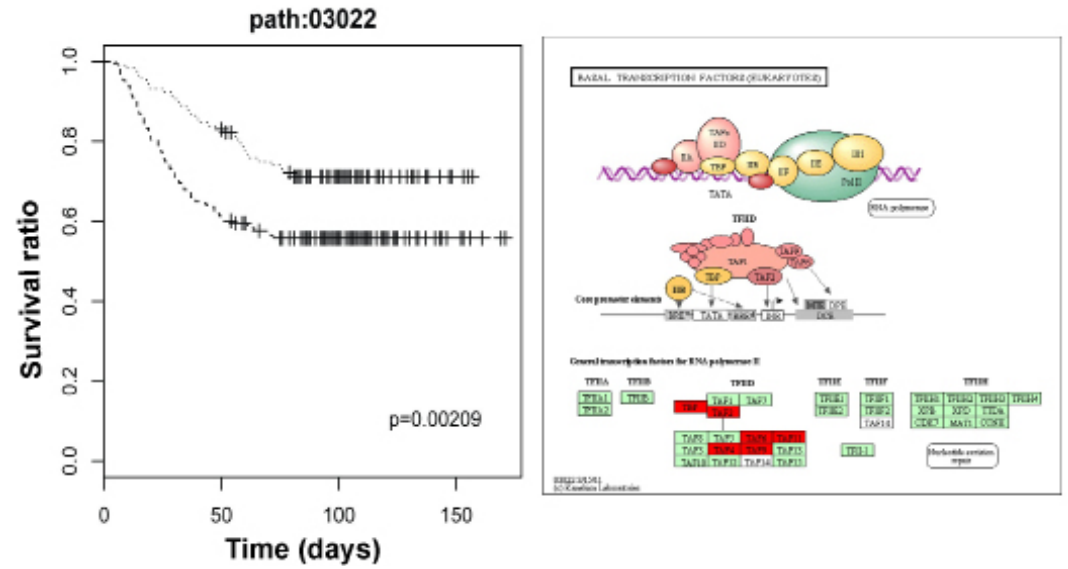

Figure 1. Survival analysis of path 03022. Left figure represents the survival curve; Right figure represents the differentially expressed genes involved in this pathway (red).

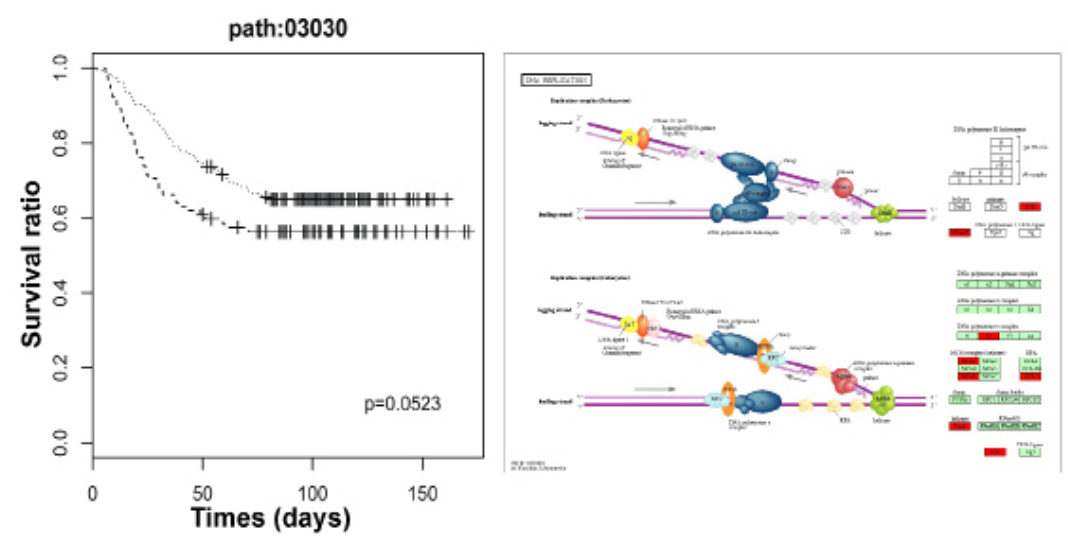

Figure 2. Survival analysis of path 03030. Left figure represents the survival curve; Right figure represents the differentially expressed genes involved in this pathway (red). 

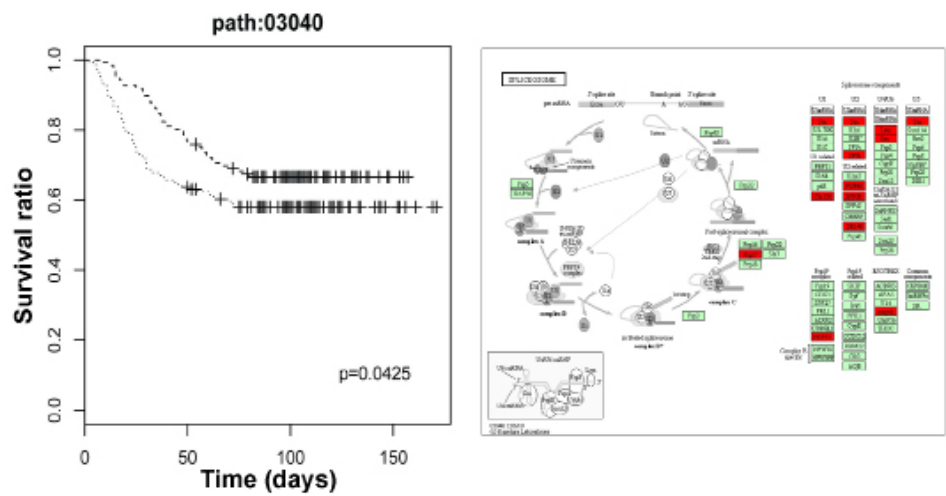

Figure 3. Survival analysis of path 03040. Left figure represents the survival curve; Right figure represents the differentially expressed genes involved in this pathway (red).
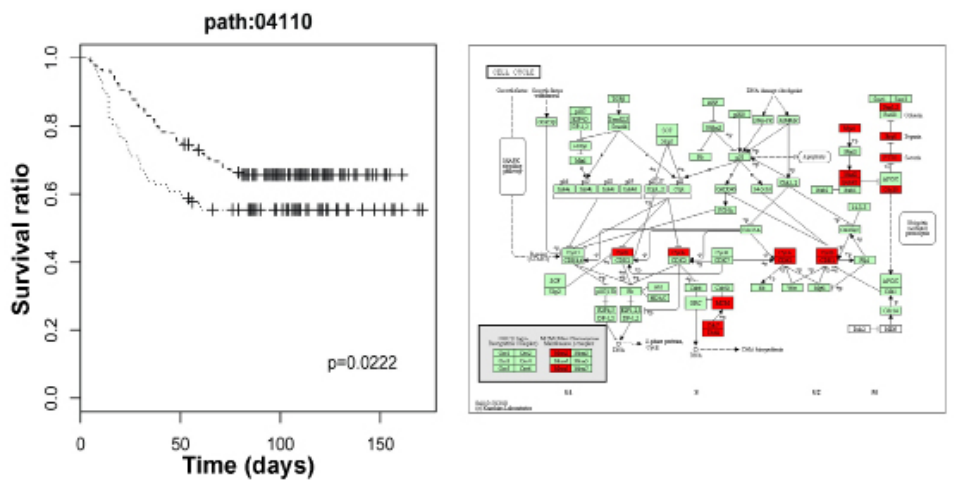

Figure 4. Survival analysis of path 04110. Left figure represents the survival curve; Right figure represents the differentially expressed genes involved in this pathway (red).

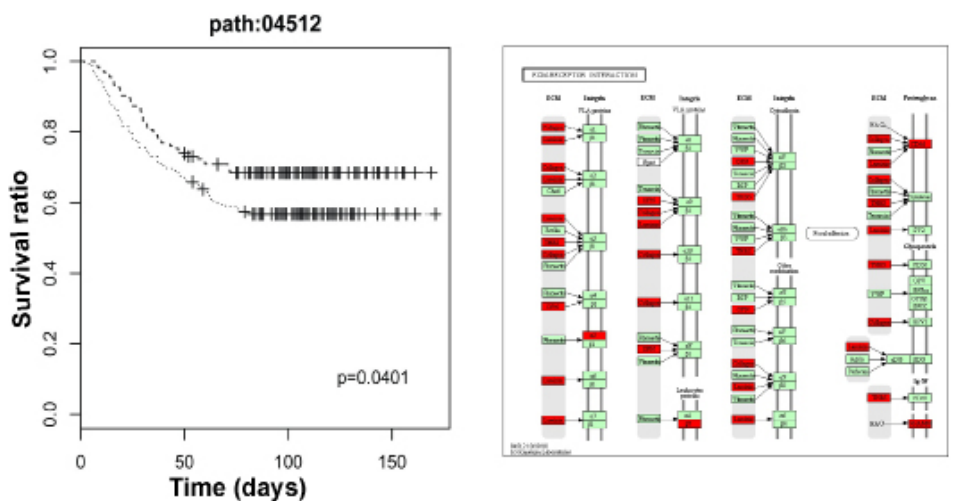

Figure 5. Survival analysis of path 04512. Left figure represents the survival curve; Right figure represents the differentially expressed genes involved in this pathway (red). 
Table 2. Results of survival analysis of pathways related with survival time of breast cancer patients.

\begin{tabular}{llc}
\hline Pathway ID & Pathway name & Survival P \\
\hline path: 00190 & Oxidative phosphorylation & 0.254 \\
path: 03022 & Basal transcription factors & $\mathbf{0 . 0 0 2 0 9}$ \\
path: 03030 & DNA replication & $\mathbf{0 . 0 5 2 3}$ \\
path: 03040 & Spliceosome & $\mathbf{0 . 0 4 2 5}$ \\
path: 03050 & Proteasome & 0.865 \\
path: 04110 & Cell cycle & $\mathbf{0 . 0 2 2 2}$ \\
path: 04512 & ECM-receptor interaction & $\mathbf{0 . 0 4 0 1}$ \\
path: 05010 & Alzheimer's disease & 0.468 \\
path: 05012 & Parkinson's disease & 0.848 \\
path: 05016 & Huntington's disease & 0.939 \\
\hline
\end{tabular}

The pathways in bold mean that $\mathrm{P}$ value is less than 0.1 .

\section{DISCUSSION}

A total of 844 DEGs in breast cancer metastasis were acquired and found to be enriched in several pathways such as the oxidative phosphorylation, DNA replication, proteasome, and ECM-receptor interaction pathways. Five pathways basal transcription factors, DNA replication, spliceosome, cell cycle and ECM-receptor interaction were related with the survival time of breast cancer recurrence.

Oxidative stress induces the secretion of matrix metalloproteinase-1, promoting the vessel growth within the tumor microenvironment (Duffy et al., 2000; Brown et al., 2000) which could increase the risk of blood-borne metastasis. p38 mitogen-associated protein kinase (MAPK) is activated by oxidative stress (Wang et al., 1998), and the phosphorylation of heat shock protein-27 by p38 MAPK has been shown to induce changes in actin dynamics (Huot et al., 1997). The proteasome, a multi-catalytic and multi-subunit protease complex, is responsible for the ubiquitin-dependent turnover of cellular proteins (Dalton, 2004; Ciechanover, 2005). Inhibition of the proteasome results in abnormal accumulation of several intracellular proteins, thereby disrupting cellular homeostasis (Codony-Servat et al., 2006) and resulting in the induction of tumor cell apoptosis (Lopes et al., 1997; An et al., 1998). The ECM modulates breast tissue homeostasis in vivo and regulates the growth, differentiation, and apoptosis of normal murine and human mammary epithelial cells in culture (BarcellosHoff et al., 1989; Petersen et al., 1992). In mammary tumors, cell-ECM interactions are disrupted whatever in vivo or in culture (Petersen et al., 1992; Bernfield et al., 1993).

Furthermore, among the 10 pathways correlated with breast cancer recurrence, 5 were found to be closely related to patient survival time according to survival analysis. Among them, basal transcription factors, a class of protein transcription factors that bind to specific sites on DNA to activate transcription and the constituents of basic transcriptional apparatus (Roeder, 1996; Dvir et al., 2001), are involved in the process of gene regulation, and sustaining life. DNA replication is the process of DNA copy for inheritance. A spliceosome, composed of small nuclear RNAs (snRNAs) and protein subunits, remove introns from a transcribed pre-mRNA (hnRNA) segment. A recent study demonstrated that overexpression of a splicing factor could trigger malignant transformation (Karni et al., 2007).

The cell cycle, or cell-division cycle, is the series of events that take place in a cell leading to its division and duplication. In this pathway, CDK1, a significant DEG, is considered to play an important role in cell proliferation and is expected to be associated with tumor 
aggressiveness and poor prognosis (Lee and Yang, 2003; Moroy and Geisen, 2004; Sutherland and Musgrove, 2004). Some recent studies have shown that CDK1 may be required for apoptosis and regulates the cell cycle (Castedo et al., 2002; Golsteyn, 2005).

ECM receptor interaction signaling pathways have been reported to be closely related with breast cancer metastasis. Laminin plays an important role in regulating cell migration and in facilitating tumor invasion (Nielsen et al., 1983). CD44 proteins participate in a large number of related molecular processes involving specific adhesions to hyaluronate, collagen and fibronectin (Lesley et al., 1993), and cell migration (Thomas et al., 1992). Type IV collagen is involved in the regulation of mammary cell proliferation, cell attachment, and migration (Kim et al., 1994).

Although the expression of some genes found to interact with DEGs was not abnormal, these genes may nonetheless transmit signals from DEGs to downstream molecules, resulting in disruptions of important functions such as the proliferation and metastasis of cancer cells.

In summary, DEGs and their corresponding signaling pathways leading to breast cancer metastasis were identified. Five of these pathways were found to be closely related to the survial time of breast cancer patients. Our results may facilitate identification of the molecular mechanisms of breast cancer and provide options for breast cancer therapy.

\section{REFERENCES}

An B, Goldfarb RH, Siman R and Dou QP (1998). Novel dipeptidyl proteasome inhibitors overcome Bcl-2 protective function and selectively accumulate the cyclin-dependent kinase inhibitor p27 and induce apoptosis in transformed, but not normal, human fibroblasts. Cell Death. Differ. 5: 1062-1075.

Barcellos-Hoff MH, Aggeler J, Ram TG and Bissell MJ (1989). Functional differentiation and alveolar morphogenesis of primary mammary cultures on reconstituted basement membrane. Development 105: 223-235.

Bernfield M, Hinkes MT and Gallo RL (1993). Developmental expression of the syndecans: possible function and regulation. Dev. Suppl. 205-212.

Brown NS, Jones A, Fujiyama C, Harris AL, et al. (2000). Thymidine phosphorylase induces carcinoma cell oxidative stress and promotes secretion of angiogenic factors. Cancer Res. 60: 6298-6302.

Castedo M, Perfettini JL, Roumier T and Kroemer G (2002). Cyclin-dependent kinase-1: linking apoptosis to cell cycle and mitotic catastrophe. Cell Death. Differ. 9: 1287-1293.

Ciechanover A (2005). Proteolysis: from the lysosome to ubiquitin and the proteasome. Nat. Rev. Mol. Cell Biol. 6: 79-87.

Claus EB, Risch N and Thompson WD (1991). Genetic analysis of breast cancer in the cancer and steroid hormone study. Am. J. Hum. Genet. 48: 232-242.

Codony-Servat J, Tapia MA, Bosch M, Oliva C, et al. (2006). Differential cellular and molecular effects of bortezomib, a proteasome inhibitor, in human breast cancer cells. Mol. Cancer Ther. 5: 665-675.

Dalton WS (2004). The proteasome. Semin. Oncol. 31: 3-9.

Duffy MJ, Maguire TM, Hill A, McDermott E, et al. (2000). Metalloproteinases: role in breast carcinogenesis, invasion and metastasis. Breast Cancer Res. 2: 252-257.

Dvir A, Conaway JW and Conaway RC (2001). Mechanism of transcription initiation and promoter escape by RNA polymerase II. Curr. Opin. Genet. Dev. 11: 209-214.

Easton DF, Ford D and Bishop DT (1995). Breast and ovarian cancer incidence in BRCA1-mutation carriers. Breast Cancer Linkage Consortium. Am. J. Hum. Genet. 56: 265-271.

Golsteyn RM (2005). Cdk1 and Cdk2 complexes (cyclin dependent kinases) in apoptosis: a role beyond the cell cycle. Cancer Lett. 217: 129-138.

Hu Z, Fan C, Oh DS, Marron JS, et al. (2006). The molecular portraits of breast tumors are conserved across microarray platforms. BMC Genomics 7: 96.

Huot J, Houle F, Marceau F and Landry J (1997). Oxidative stress-induced actin reorganization mediated by the p38 mitogen-activated protein kinase/heat shock protein 27 pathway in vascular endothelial cells. Circ. Res. 80: 383-392.

Kamangar F, Dores GM and Anderson WF (2006). Patterns of cancer incidence, mortality, and prevalence across five continents: defining priorities to reduce cancer disparities in different geographic regions of the world. J. Clin. Oncol. 24: $2137-2150$ 
Kanehisa M and Goto S (2000). KEGG: kyoto encyclopedia of genes and genomes. Nucleic Acids Res. 28: 27-30.

Karni R, de Stanchina E, Lowe SW, Sinha R, et al. (2007). The gene encoding the splicing factor SF2/ASF is a protooncogene. Nat. Struct. Mol. Biol. 14: 185-193.

Kim JP, Chen JD, Wilke MS, Schall TJ, et al. (1994). Human keratinocyte migration on type IV collagen. Roles of heparin-binding site and alpha 2 beta 1 integrin. Lab. Invest. 71: 401-408.

Lee MH and Yang HY (2003). Regulators of G1 cyclin-dependent kinases and cancers. Cancer Metastasis Rev. 22: 435 449.

Lesley J, Hyman R and Kincade PW (1993). CD44 and its interaction with extracellular matrix. Adv. Immunol. 54: 271335.

Li C, Li X, Miao Y, Wang Q, et al. (2009). SubpathwayMiner: a software package for flexible identification of pathways. Nucleic Acids Res. 37: e131.

Lopes UG, Erhardt P, Yao R and Cooper GM (1997). p53-dependent induction of apoptosis by proteasome inhibitors. $J$. Biol. Chem. 272: 12893-12896.

Moroy T and Geisen C (2004). Cyclin E. Int. J. Biochem. Cell Biol. 36: 1424-1439.

Nielsen M, Christensen L and Albrechtsen R (1983). The basement membrane component laminin in breast carcinomas and axillary lymph node metastases. Acta Pathol. Microbiol. Immunol. Scand. A 91: 257-264.

Olopade OI, Grushko TA, Nanda R and Huo D (2008). Advances in breast cancer: pathways to personalized medicine. Clin. Cancer Res. 14: 7988-7999.

Perou CM, Sorlie T, Eisen MB, van de Rijn M, et al. (2000). Molecular portraits of human breast tumours. Nature 406: 747-752.

Petersen OW, Ronnov-Jessen L, Howlett AR and Bissell MJ (1992). Interaction with basement membrane serves to rapidly distinguish growth and differentiation pattern of normal and malignant human breast epithelial cells. Proc. Natl. Acad. Sci. U. S. A. 89: 9064-9068.

Roeder RG (1996). The role of general initiation factors in transcription by RNA polymerase II. Trends Biochem. Sci. 21: 327-335.

Sarasin A and Kauffmann A (2008). Overexpression of DNA repair genes is associated with metastasis: a new hypothesis. Mutat. Res. 659: 49-55.

Schubert EL, Mefford HC, Dann JL, Argonza RH, et al. (1997). BRCA1 and BRCA2 mutations in Ashkenazi Jewish families with breast and ovarian cancer. Genet. Test. 1: 41-46.

Sorlie T, Perou CM, Tibshirani R, Aas T, et al. (2001). Gene expression patterns of breast carcinomas distinguish tumor subclasses with clinical implications. Proc. Natl. Acad. Sci. U. S. A. 98: 10869-10874.

Sorlie T, Wang Y, Xiao C, Johnsen H, et al. (2006). Distinct molecular mechanisms underlying clinically relevant subtypes of breast cancer: gene expression analyses across three different platforms. BMC Genomics 7: 127.

Sutherland RL and Musgrove EA (2004). Cyclins and breast cancer. J. Mammary. Gland. Biol. Neoplasia 9: 95-104.

Thomas L, Byers HR, Vink J and Stamenkovic I (1992). CD44H regulates tumor cell migration on hyaluronate-coated substrate. J. Cell Biol. 118: 971-977.

Wang X, Martindale JL, Liu Y and Holbrook NJ (1998). The cellular response to oxidative stress: influences of mitogenactivated protein kinase signalling pathways on cell survival. Biochem. J. 333: 291-300.

Wang Y, Klijn JG, Zhang Y, Sieuwerts AM, et al. (2005). Gene-expression profiles to predict distant metastasis of lymphnode-negative primary breast cancer. Lancet 365: 671-679.

Yu K, Lee CH, Tan PH and Tan P (2004). Conservation of breast cancer molecular subtypes and transcriptional patterns of tumor progression across distinct ethnic populations. Clin. Cancer Res. 10: 5508-5517. 